\title{
A New Judgment Method on Ship Accident of Waterway
}

\author{
Shukui Zhang ${ }^{1,}$, Jing $\mathrm{Li}^{1, \mathrm{~b}}$, Xiangong Yan ${ }^{1, \mathrm{c}}$ \\ 1 Jiangsu Maritime Institute Jiangsu Nanjing 211170, China. \\ azhangshkfy@163.com, b158142476@qq.com, c337074997@163.com
}

\begin{abstract}
According to the shortcomings of judgment method on traffic accident in waterway, the full Bayesian-based potential for safety improvement method is built in the article. The hot-segment of accident is identified with the difference between accident number of a segment waterway and accident expectation of entire waterway. The new identification method is used to identify hotsegment of accident of the Yangtze River downstream and which proves that the new identification is better. At last, three kinds of evaluation of SCT (site consistency test), MCT (method consistency test) and TRDT (total rank differences test) are used to evaluate the identification methods and the new identification method is further proved the best.
\end{abstract}

Keywords: Judgment, Bayesian estimation, negative binomial model, potential for safety improvement.

\section{Introduction}

The fairway waters are the places where ship traffic accidents frequently occur. Traditional methods of traffic accident prediction are used in navigation channels with large flow rates of ships, and there is little room for improvement in navigation safety of these channels. Studies have shown that the occurrence of traffic accidents is caused by the characteristics of the waterway, the reduction of accident rate can be achieved by improving the characteristics of the waterway. Recently, the potential for safety improvement method based on full Bayesian security has become a research hotspot, the definition is the difference between the absolute number and the expectations of traffic accidents, and the greater the difference is, and the more potential there is for the reduction of accidents, so there is greater potential for safety improvement.

A negative binomial model is constructed in the paper and the Bayesian potential for safety improvement method is used to identify waterway which accident occurs easily. Finally, the judging results are compared with the results of full Bayesian method, empirical Bayed method and absolute number of accident methods.

\section{Data Sources}

12 key waterways in Jiangsu section of Yangtze River were selected as the research object, according to the characteristics of theirs, 11 variables were collected and selected for safety analysis, at the same time, traffic accidents data of 12 waterway occurred in 2015 and 2016 were collected, among them, data of 2015 was used to construct accident prediction model and data of 2016 was used to calculate evaluation indicators of various judging methods. The relevant statistical values of 11 independent variables are shown in Table 1.

\section{Bayesian Potential for Safety Improvement}

\subsection{Empirical Bayesian Method}

Empirical Bayesian method uses a linear combination of multiple Gaussian functions to represent the probability density function of the data, by determining the weights of each Gaussian function and combining the occurred traffic accident data with the expected values of whole channel traffic accidents obtained by using the regression model, then the number of traffic accident that will occur in the waterway is estimated. The calculation method is shown in equation (1). 


$$
\eta_{\mathrm{BE}, k}=\eta_{\mathrm{BN}, k}\left(1+\frac{\eta_{\mathrm{BN}, k}}{\delta}\right)^{-1}+x_{k}\left(1-\left(1+\frac{\eta_{\mathrm{BN}, k}}{\delta}\right)^{-1}\right)
$$

Tab 1. Descriptive statistics for variables of segment waterway

\begin{tabular}{|c|c|c|c|}
\hline category & waterway variables & $\begin{array}{l}\text { Average } \\
\text { value }\end{array}$ & $\begin{array}{l}\text { standard } \\
\text { deviation }\end{array}$ \\
\hline \multirow{4}{*}{$\begin{array}{c}\text { geometric } \\
\text { characteristics }\end{array}$} & Length(Total length of waterway $/ \mathrm{km}$ ) & 26.46 & 9.24 \\
\hline & Depth(waterway average depth/m) & 15.76 & 4.35 \\
\hline & Width(waterway average width/m) & 1250 & 72.42 \\
\hline & Number of turning points & 4.15 & 0.84 \\
\hline \multirow{4}{*}{$\begin{array}{c}\text { traffic } \\
\text { characteristics }\end{array}$} & Saturation(ratio of traffic volume to navigation capacity) & 84.54 & 18.36 \\
\hline & daily average flow(thousands ship) & 2.05 & 0.27 \\
\hline & $\begin{array}{l}\text { ship speed difference(speed difference between two } \\
\text { adjacent vessels with the same way) }\end{array}$ & 4.65 & 2.87 \\
\hline & $\begin{array}{l}\text { ship speed change rate(vessel speed change per unit time / } \\
\mathrm{m} / \mathrm{s} 2)\end{array}$ & 0.14 & 0.04 \\
\hline \multirow{3}{*}{$\begin{array}{l}\text { environmental } \\
\text { factors }\end{array}$} & $\begin{array}{c}\text { Visibility(standard days that can seeing less than } 2 \mathrm{~km} \\
\text { every year) }\end{array}$ & 100.25 & 28.42 \\
\hline & $\begin{array}{l}\text { strong wind(standard days that winds above grade } 7 \text { per } \\
\text { year) }\end{array}$ & 57.65 & 10.26 \\
\hline & maximum water speed & 3.12 & 0.72 \\
\hline
\end{tabular}

Here $\eta_{\mathrm{BE}, k}$ denotes the estimated expected value of the $\mathrm{k}$-th waterway traffic accident using the empirical Bayesian method; $\eta_{\mathrm{BN}, k}$ denotes the estimated expected value of the $\mathrm{k}$-th waterway traffic accident using the Negative binomial model; $x_{k}$ is the number of actual traffic accidents occurred in the k-th waterway; $\delta$ is over dispersion coefficient, its value is $\frac{1}{\gamma} ; \gamma$ is Negative Binomial Model's discrete coefficient.

\subsection{Full Bayesian Method}

Full Bayesian method is to set a specific distribution for each parameter's prior distribution first, and then obtains the posterior distribution of each parameter according to actual ship accident data. The calculating method can be expressed as follows:

$$
\omega(\theta \mid x)=\frac{L(x \mid \theta) \omega(\theta)}{\int L(x \mid \theta) \omega(\theta) \mathrm{d} \theta}
$$

Here $\theta$ is expected value of ship traffic accidents; $x$ is the number of actual traffic accidents; $L(x \mid \theta)$ is likelihood function; $\omega(\theta)$ is prior distribution of $\theta$ and $\omega(\theta \mid x)$ is its posterior distribution; $\int L(x \mid \theta) \omega(\theta) \mathrm{d} \theta$ is marginal probability distribution of actual traffic accident data.

Based on Bayesian theory, the negative binomial model is built in the paper and its expression is as follows:

$$
\begin{gathered}
x_{k} \sim \operatorname{Negbin}\left(\eta_{k}, \gamma\right) \\
\operatorname{Ln} \eta_{\mathrm{BF}, k}=\alpha_{0}+\sum_{q=1}^{n} \alpha_{q} Y_{q k}
\end{gathered}
$$

Here $x_{k}$ is the number of actual traffic accidents of the k-th waterway; $\eta_{\mathrm{BF}, k}$ is expected value of the k-th waterway traffic accident obtained the Negative binomial model based on the full Bayesian method; $\alpha$ is variable coefficient vector used for channel safety analysis; $\alpha_{q}$ is variable 
coefficient of the qth; $Y_{q k}$ is independent vector; $\mathrm{n}$ is number of variables used for waterway safety analysis.

\subsection{Potential for Safety Improvement}

Potential for safety improvement means the section that the number of traffic accidents estimated using Bayesian is exceed the average number of traffic accidents of the whole waterway. Its calculating method is as follows:

$$
S_{\mathrm{BF}, k}=\eta_{\mathrm{BF}, k}-\eta_{\mathrm{BN}, k}
$$

\section{Comparison of Traffic Accidents in Different Waterway}

\subsection{Evaluation Method Selection}

Three evaluation methods are used as following in the paper:

(1)SCT (site consistency test) the calculation method is as follows:

$$
P_{\mathrm{SC}, j}=\frac{\sum_{i=n-n r+1}^{n} A_{i, j, k+1}}{\sum_{i=n-n r+1}^{n} T_{i, j, k+1}}
$$

Here $\mathrm{j}$ is different judging methods; $\mathrm{i}$ is traffic accident ranking; $\mathrm{n}$ is total number of waterway; $\mathrm{r}$ is percentage of waterway that are judged as traffic accidents often occur; $A_{i, j, k+1}$ is the number of traffic accidents that occurred in the $\mathrm{k}+1$ year of waterways with traffic accident ranked $\mathrm{i}$, in this article it refers to the number of traffic accidents in 2016; $T_{i, j, k+1}$ is total ship traffic volume in the year $\mathrm{k}+1$ of waterways with traffic accidents ranked $\mathrm{i}$.

(2)MCT (method consistency test) the calculation method is as follows:

$$
P_{\mathrm{MC}, j}=\left\{i_{n-n r r+1}, i_{n-n r}, \mathrm{~L}, i_{n}\right\}_{j, k} \mathrm{I} \quad\left\{i_{n-n r+1}, i_{n-n r}, \Lambda, i_{n}\right\}_{j, k+1}
$$

Here $\mathrm{k}$ is the first year and $\mathrm{k}+1$ is the second year; in is the waterway that ranks first in traffic accidents. If no measures are taken in the second year to improve the safety of accidental waterway, and the characteristics of waterway do not change significantly within two consecutive years, then judging results in two consecutive years with better judgment method should be closer.

(3) TRDT(total rank differences test).This method determines which one is better by using difference of ranking of traffic accidents within two consecutive years. The calculation method is as follows:

$$
P_{\mathrm{TRD}, j}=\sum_{i=n-n r+1}^{n}\left|R\left(i_{j, k}\right)-R\left(i_{j, k+1}\right)\right|
$$

Here $R\left(i_{j, k}\right)$ denotes traffic accident ranking of a waterway in the kth year; $R\left(i_{j, k+1}\right)$ denotes traffic accident ranking of a waterway in the $k+1$ th year.

\subsection{Comparison of Various Judgment Results}

Assuming that waterways whose rank are the top 4, top 7, and top 10 are waterways which occur traffic accidents easily, then the value of SCT, MCT, and TRDT of each judgment method are calculated by using traffic accident data of 2016. The results are shown in Table 3. 
Tab 2. Comparison of 3 evaluation criterion on 4 methods

\begin{tabular}{|c|c|c|c|c|c|c|c|c|c|}
\hline \multirow{2}{*}{ judgment method } & \multicolumn{9}{|c|}{ judgment standard } \\
\cline { 2 - 12 } & \multicolumn{3}{|c|}{ SCT } & \multicolumn{3}{c|}{ MCT } & \multicolumn{3}{c|}{ TRDT } \\
\cline { 2 - 11 } & top 4 & top 7 & top 10 & top 4 & top 7 & top 10 & top 4 & top7 & top 10 \\
\hline $\begin{array}{c}\text { absolute number of } \\
\text { accidents }\end{array}$ & 0.62 & 0.54 & 0.62 & 1 & 2 & 3 & 12 & 19 & 25 \\
\hline experience Bayesian & 0.62 & 0.55 & 0.63 & 2 & 3 & 4 & 13 & 18 & 25 \\
\hline full Bayesian & 0.74 & 0.70 & 0.71 & 3 & 5 & 6 & 6 & 11 & 15 \\
\hline $\begin{array}{c}\text { potential for safety } \\
\text { improvement }\end{array}$ & 0.76 & 0.71 & 0.72 & 4 & 6 & 8 & 4 & 7 & 13 \\
\hline
\end{tabular}

SCT: Under the condition of determination of waterways which occur traffic accidents easily, full Bayesian method and full Bayesian potential for safety improvement method are obviously better than empirical Bayesian method and absolute accident number method, besides, potential for safety improvement is better than full Bayesian method.

MCT: The ranking in turn are full Bayesian potential for safety improvement, full Bayesian, empirical Bayesian and absolute number of accidents: the MCT values of full Bayesian potential for safety improvement are 4, 6 and 8 .

TRDT: Full Bayesian and full Bayesian potential for safety improvement are better than other two methods, here full Bayesian potential for safety improvement is best, and its value is 13 .

\section{Conclusion}

The full Bayesian potential for safety improvement is not only avoid random volatility defects of actual accident data and the deficiency of traditional Bayesian method on parameter estimation, but also makes it easier to identify waterways with higher traffic accident reduction potential. The conclusions have important reference value for waterway which occur traffic accidents easily.

\section{References}

[1]. Huang H, Chin H C, Hague M. Empirical evaluation of alternative approaches in identifying crash hotpots: Naive ranking, empirical bays and full bays [J]. Transport Research Record: Journal of the Transportation Research Board, 2010, 2013(5):32--38.

[2]. Land B, Persuade B. Fully Bayesian approach to investigate and evaluate ranking criteria for black spot identification [J]. Transportation Research Record: Journal of the Transportation Research Board, 2011, 2247(13):105--112.

[3]. Xin K, Wang X S, Chen X H. Corridor-level signalized intersection safety analysis in Shanghai: China using Bayesian hierarchical models [J]. Accident Analysis and Prevention, 2013, 50(1): 25-31.

[4]. Goo F, Wang X, Abdel-Arty M. Modeling signalized intersection safety with corridor-level spatial correlations [J]. Accident Analysis and Prevention, 2010, 42(1): 84-89.

[5]. Hayashi Ichihara Sadamitsu, Enema Yogi. Development of Counting Method of Black Spots Caused by the Radioactive Contaminations on the Imaging Plate [J]. Nihon Hoarsen Jujutsu Sasha, 2012, 68(5): 45-53. 